\title{
RESET
}

Recherches en sciences sociales sur Internet

\section{La stratification sociale des pratiques numériques des adolescents}

The Social Stratification of Digital Practices Among Teenagers

\section{Pierre Mercklé et Sylvie Octobre}

\section{(2) OpenEdition}

\section{Journals}

Édition électronique

URL : https://journals.openedition.org/reset/129

ISSN : 2264-6221

\section{Éditeur}

Association Recherches en sciences sociales sur Internet

\section{Référence électronique}

Pierre Mercklé et Sylvie Octobre, «La stratification sociale des pratiques numériques des adolescents », RESET [En ligne], 1 | 2012, mis en ligne le 30 décembre 2012, consulté le 21 janvier 2023. URL : http://journals.openedition.org/reset/129

Ce document a été généré automatiquement le 21 janvier 2023.

Creative Commons - Attribution - Pas d'Utilisation Commerciale - Partage dans les Mêmes Conditions 4.0 International - CC BY-NC-SA 4.0

https://creativecommons.org/licenses/by-nc-sa/4.0/ 


\title{
La stratification sociale des pratiques numériques des adolescents
}

The Social Stratification of Digital Practices Among Teenagers

\author{
Pierre Mercklé et Sylvie Octobre
}

1 La tradition sociologique est maintenant ancienne (Bourdieu, 1979; Donnat \& Cogneau, 1990 ; Donnat, 1998, 2009), qui consiste à la fois à décrire les degrés et les formes des investissements des Français en matière de participation culturelle, et à les rapporter à certaines de leurs caractéristiques socio-démographiques (catégorie socioprofessionnelle, niveau de diplôme, sexe, âge...) pour mesurer les niveaux des inégalités sociales en matière d'accès à la culture ${ }^{1}$. Destinées à mesurer les effets des politiques publiques en matière de démocratisation culturelle, les grandes enquêtes issues de cette tradition de sociologie critique ont en réalité surtout démontré la persistance d'une forte stratification sociale des pratiques culturelles, que celles-ci soient considérées dans leur ensemble (Donnat, 1999), ou qu'elles portent sur des pratiques spécifiques, comme par exemple la fréquentation des musées (Lehalle \& Mironer, 1993). Plus de trente après la parution de La Distinction, ces enquêtes continuent de témoigner $\mathrm{du}$ maintien de relations étroites entre position sociale d'une part, et dotation en équipements, détentions de compétences, intensités d'investissement dans les loisirs culturels, usages et goûts d'autre part.

2 La "révolution numérique ", si elle a profondément changé les usages en matière de culture et de loisirs, ne semble pas toutefois avoir complètement bouleversé les principes de cette stratification sociale des pratiques culturelles: malgré l'apparente généralisation des technologies numériques, d'importantes inégalités subsistent en effet en matière aussi bien d'équipement des foyers en ordinateurs, que d'accès et d'usages de l'Internet (Donnat, 2009). Ce constat établi à partir d'une observation des pratiques culturelles des adultes ${ }^{2}$, vaut-il également pour les adolescents? Qu'en est-il, en matière de stratification sociale des pratiques numériques, de ceux qui ont été enfants et adolescents dans les années 2000, et qu'on a parfois appelé les «digital 
natives» (Prensky, 2001) ? Quelles sont les mutations culturelles et sociales portées par ces jeunes générations?

3 Après avoir longtemps été négligées par la sociologie de la culture, comme si elles avaient été implicitement considérées soit comme des reproductions des pratiques parentales, soit comme les résultats de consommations passives soumises aux prescriptions des industries culturelles de masse, les pratiques et les préférences des adolescents en matière de loisirs culturels en général (Octobre, 2004) et d'usages numériques en particulier (Buckingham, 2000 ; Watkins, 2009; Metton-Gayon, 2009) commencent à faire l'objet d'un certain nombre d'investigations et d'analyses. Dans leur prolongement, l'enquête longitudinale sur les pratiques culturelles et de loisirs des enfants et des adolescents réalisée à l'initiative du Ministère de la Culture (Octobre, Détrez, Mercklé \& Berthomier, 2010) se distingue par son ampleur et sa systématicité : elle porte sur 3900 enfants, entrés en cours préparatoire (CP) en 1997 et suivis jusqu'au milieu des années de lycée, qui ont donc été adolescents dans la décennie 2000, entre la généralisation du téléphone portable et celle des réseaux sociaux en ligne (Mercklé, 2011 : 73-95). Ces enfants ont été interrogés tous les deux ans par questionnaire sur l'ensemble de leurs loisirs, de leurs pratiques culturelles et de leurs goûts, et cela donc quatre fois successivement entre 2002 et 2008 (autrement dit à 11, 13, 15 et 17 ans).

4 Au sein de cette vaste enquête qui vient de s'achever, les pratiques numériques des adolescents occupent évidemment une place importante: ces derniers ont été interrogés sur leurs équipements, les fréquences de leurs pratiques, leurs usages et leurs préférences en matière de jeux vidéo, et surtout d'utilisation de l'ordinateur et du téléphone portable ${ }^{3}$. Les données collectées permettent de mieux connaitre les univers culturels de cette génération et leurs évolutions au fil de l'avancée en âge, mais également de mesurer le poids des diverses transmissions (des parents, des fratries, des copains, de l'école), dans un domaine marqué par le renouvellement technologique, qui pourrait sembler échapper par nature aux transmissions de long terme, du moins au sens traditionnel. Ces observations permettent de repenser la stratification sociale des pratiques d'une part en intégrant à l'analyse les effets des mutations conjoncturelles et de l'autre, en précisant les modalités conjointes de divers facteurs de clivages. Dès lors que les différences d'accès sont observées sur un plan chronologique, on peut dénouer les effets de l'origine sociale de ceux du genre à travers les calendriers différentiels d'accès, d'usages et d'attachement au numérique.

\section{Les pratiques numériques, facteur de convergence générationnelle}

5 L'adolescence se caractérise par une réorganisation des agendas culturels, qui prend une double forme: celle d'une redéfinition des répertoires de loisirs, et celle d'une modification des rythmes de chaque activité. Cette réorganisation prend appui sur les mutations des cadres temporels des enfants, qui passent d'emplois du temps stables à l'école primaire à une flexibilité plus grande au collège et au lycée, et sur l'augmentation tendancielle à la fois du temps passé sans la présence d'un adulte, mais aussi des pressions qui s'exercent sur eux, aussi bien scolaires (avec notamment l'augmentation de la charge de travail) qu'extra-scolaires (liées à la montée des injonctions à la sociabilité amicale, par exemple). La réorganisation des agendas matérialise également le changement de statut de l'enfant au sein de la famille sur 
plusieurs registres: intérieur/extérieur, avec la peur éventuelle de l'extérieur et le désir de protection de l'enfant qui favorise l'émergence de la culture de la chambre (Glévarec, 2010), mais également l'apprentissage de cet extérieur par l'acquisition progressive de l'autonomie à distance permise par le numérique ; travail/loisir, avec la transition du contrôle au libre-arbitre; famille/copains, avec le basculement des centres de la gravité identitaire (Pasquier, 2005). Dans ces différents registres, le numérique a pris une importance considérable, à tel point qu'il peut apparaître aujourd'hui comme central dans les expériences culturelles à l'adolescence.

\section{Le numérique au cœur des univers culturels}

6 Les adolescents grandissent désormais dans un monde où le numérique est omniprésent. Que l'on parle de "digital natives », de " génération Y » (Dagnaud, 2011) ou de «troisième âge médiatique » (Donnat, 2009), les terminologies, des plus discutables aux plus heuristiques, disent toutes cette centralité du numérique dans les rapports des jeunes générations à la culture. L'observation des évolutions avec l'avancée en âge des univers culturels de celles et ceux qui ont été adolescent-e-s dans les années 2000 en témoigne : le temps de leur enfance était encore celui des anciens médias, tandis que leur adolescence a été celui du basculement vers les nouveaux écrans, que l'on considère la fréquence de la pratique quotidienne ou les niveaux d'attachement (Figure 1). Jusqu'à 15 ans, la corrélation entre pratique et attachement reste globalement négative : la télévision, regardée quotidiennement de façon massive, est la pratique qui suscite le plus faible attachement, et à l'inverse le sport (plutôt pour les garçons) et les pratiques artistiques (plutôt pour les filles) combinent fort attachement et faible pénétration de la pratique quotidienne. Mais à partir de 15 ans, apparaît une combinaison auparavant inédite entre attachement et pratique quotidienne, et elle concerne les deux activités désormais emblématiques des univers culturels des adolescents, à savoir l'écoute de musique et l'utilisation de l'ordinateur.

Télévision, radio, musique, lecture de livres, de journaux et de magazines, de bandes dessinées, sport, jeux vidéo, autres jeux, ordinateur, pratiques artistiques et écriture d'un journal intime... L'éventail des pratiques est large, mais si la moyenne du nombre d'activités pratiquées quotidiennement reste stable, elle masque néanmoins deux tendances inverses : une baisse de la proportion de ceux qui font beaucoup de pratiques quotidiennes (plus de cinq activités) ainsi que de ceux qui en font peu (moins de trois pratiques), de plus en plus d'enfants avec l'avancée en âge exerçant quotidiennement un nombre moyen d'activités (entre trois et cinq activités).

8 C'est donc plutôt une normalisation des comportements que l'on observe, dans laquelle le numérique occupe une place de plus en plus centrale. L'ordinateur devient en effet au fil du temps un objet incontournable, sans que l'on puisse vraiment distinguer ici l'effet de l'âge ou de la généralisation de l'équipement, voire du multi-équipement, liée notamment à la baisse manifeste des coûts en quelques années (Maresca, Picard \& Pilorin, 2011). Entre le début et la fin du collège, la part des enfants qui ne disposaient pas d'un ordinateur à domicile a diminué d'un tiers, et plus d'un sur cinq possède alors un ordinateur à titre personnel. Ils sont plus nombreux encore au lycée puisque plus d'un sur trois possède un ordinateur. Et si $14,5 \%$ des enfants de 11 ans déclaraient se servir de l'ordinateur tous les jours, ils sont $69 \%$ six ans plus tard à en avoir un usage quotidien. La pratique devient d'ailleurs de plus en plus autonome, corrélativement 
sans doute à la possession d'un ordinateur personnel : si un enfant sur dix se sert d'un ordinateur avec un de ses parents à 11 ans, ils ne sont plus que $3 \%$ à 17 ans (et respectivement $26 \%$ puis $11,3 \%$ avec des frères et sœurs). Ces résultats attestent de l'ampleur et de la rapidité de cette révolution numérique au sein de leur génération.

Figure : Cartographie des univers culturels
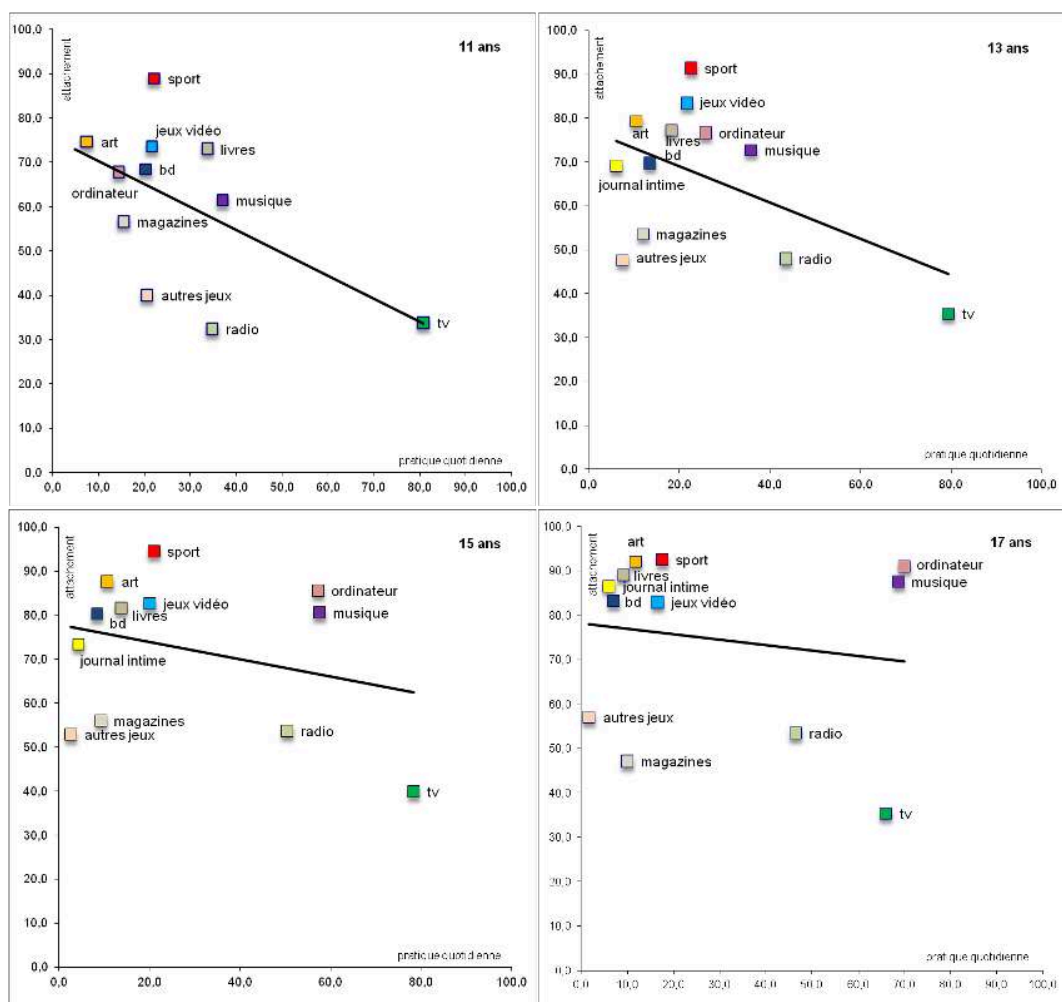

Base : tous les enfants.

NB : en abscisses figurent les proportions d'adolescents qui effectuent la pratique tous les jours ou presque et en ordonnées la proportion de ces pratiquants quotidiens qui se déclarent attachés à la pratique. Le premier graphique (en haut à gauche) concerne les enfants à 11 ans, le deuxième (en haut à droite) les enfants à 13 ans, le troisième (en bas à gauche), les enfants à 15 ans et le quatrième (en bas à droite), les enfants à 17 ans.

Note de lecture : à 11 ans, $81 \%$ des enfants regardent la télévision tous les jours, mais seulement 34\% d'entre ceux-ci, déclarent que ça leur manquerait beaucoup s'ils ne pouvaient plus le faire. La droite située sur chaque graphique est la droite de régression linéaire permettant de représenter graphiquement la relation entre attachement et pratique.

\section{La diversité des usages}

Que font les adolescents avec l'ordinateur? L'observation de l'évolution des usages saisis par l'enquête permet de se convaincre de l'importance croissante de la nature hyper-médiatique de ces usages : en début de collège, l'usage majoritaire est le jeu (jeux vidéo : $73 \%$ ), loin devant les usages communicationnels (messagerie : $33 \%$; forums et chats : $20,5 \%$ ) et la consommation de produits culturels (consultations de CD-ROM : $29 \%$; téléchargement de musique, de DVD, films ou DivX : $23 \%$ ). Avec le passage en seconde moitié de collège, les usages se diversifient et deviennent plus fréquents, à l'exception des jeux vidéo: les usages communicationnels se démultiplient (courrier électronique : $57 \%$; messagerie instantanée : $75 \%$; forums et chats : $22 \%)^{4}$; la consommation culturelle prend une place importante (celle de musique est multipliée 
par 2, et celle de films, quasi-inexistante auparavant, concerne désormais 39,5\% des adolescents qui utilisent un ordinateur), et s'accompagne d'un développement de la création (dessins, photos $33 \%$ ), quand, dans le même temps, la pratique «traditionnelle » de ces mêmes activités est en déclin. Par ailleurs, l'accroissement de la pression scolaire favorise l'augmentation des recherches sur Internet (56\% en début de collège, $79 \%$ au lycée), nouvelle encyclopédie interactive du savoir. L'ordinateur joue ainsi de la porosité des registres, éducatif et ludique : il s'impose tout à la fois comme mode d'accès au savoir et outil de travail scolaire (la part de ceux qui utilisent l'ordinateur pour faire leurs devoirs est multipliée par 25 avec le passage en seconde moitié de collège), mais aussi comme porte ouverte sur l'ensemble des centres d'intérêts des adolescents, au premier rang desquels figurent la sociabilité amicale et la conversation avec les pairs. Ainsi, dès le début du collège, les usages mixtes (ludiques et scolaires) de l'ordinateur sont devenus majoritaires (plus de 6 adolescents sur 10).

La multiplicité des usages est-elle seulement le produit du nombre de plus en plus important d'usagers, ou chaque usager présente-t-il lui-même aussi un éventail plus diversifié d'usages qu'auparavant? Comment ceux-ci évoluent-ils avec l'avancée en âge ? Le dénombrement des usages déclarés par chaque enquêté révèle une très grande variété, qu'on la mesure par le nombre moyen d'usages différents, ou par la proportion d'adolescents qui déclarent au moins trois usages différents de l'ordinateur (Tableau 1). La multiplicité des usages ne fait que croître avec l'avancée en âge, et particulièrement rapidement en seconde moitié de collège. Il est malaisé de démêler ici les parts respectives de l'effet d'âge et de l'effet de période : est-ce parce que les adolescents ont grandi que leurs usages de l'ordinateur se sont diversifiés? Ou bien est-ce en réalité que le champ des possibles, marqué par l'explosion des potentialités multimédias de l'informatique domestique, s'est profondément diversifié précisément à ce moment-là ?

Tableau : Diversité des usages de l'ordinateur et avancée en âge (\%)

\begin{tabular}{|l|l|l|l|l|}
\hline Nombre d'usages de l'ordinateur & $\mathbf{1 1}$ ans & $\mathbf{1 3}$ ans & $\mathbf{1 5}$ ans & $\mathbf{1 7}$ ans \\
\hline Aucun & 3 & 2 & 2 & 4 \\
\hline Un & 25 & 21 & 6 & 3 \\
\hline Deux & 24 & 29 & 10 & 6 \\
\hline Trois & 23 & 22 & 14 & 11 \\
\hline Quatre & 14 & 15 & 17 & 18 \\
\hline Cinq et plus & 11 & 11 & 51 & 58 \\
\hline Ensemble & 100 & 100 & 100 & 100 \\
\hline Nombre moyen d'usages & 2,6 & 2,7 & 4,6 & 4,9 \\
\hline
\end{tabular}

Base : enfants qui utilisent un ordinateur au moins une fois par mois. Note de lecture : Parmi les enfants de 11 ans qui utilisent l'ordinateur au moins une fois par mois, $25 \%$ déclarent en avoir un seul usage, et $24 \%$ deux usages différents. 
11 Une enquête menée en 2010 sur les usages d'Internet, auprès d'enfants de primaire, de collège et de lycée, permet de préciser l'analyse (Kredens \& Fontar, 2010) : selon cette enquête, les enfants de primaire pratiquent principalement quatre activités (jeux, écoute de musique, visionnage de vidéos et recherches personnelles), les collégiens en pratiquent neuf (les mêmes, plus : discussions en ligne, recherches scolaires, mails, consultation de blogs de tiers, téléchargement), et les lycéens, onze (celles des collégiens, plus: consultation d'actualités, et achats). A la diversité des usages correspond la multiplicité des sites consultés : toujours selon cette enquête, derrière les incontournables du Net, tels que Facebook, YouTube ou MSN (donnés comme sites préférés par respectivement $28 \%, 15,2 \%$ et $8,3 \%$ des jeunes interrogés), 338 sites différents sont listés comme sites préférés. Culture commune donc, mais qui n'empêche pas une personnalisation des goûts.

\section{Le régime de valeur du numérique, facteur de convergence générationnel}

12 L'installation du numérique au cœur des univers culturels adolescents n'est pas seulement un changement de pratiques ou de supports. En réalité, il met en jeu un nouveau régime de valeur culturelle (Frow, 1995), celui d'un troisième âge médiatique (Donnat, 2009) ou d'un âge "hyper-médiatique ", caractérisé par plusieurs lignes de mutation fortes des rapports à la culture chez les adolescents de cette génération.

13 Tout d'abord, le numérique rompt chez eux l'association classique entre pratique et support: l'ordinateur peut servir à écouter de la musique, regarder un film, écrire, dessiner, composer, discuter, faire des recherches, etc. La même remarque peut être faite pour les téléphones portables qui servent certes à téléphoner, mais également à prendre des photos (pour 75,5\% des possesseurs de 17 ans), à écouter de la musique $(65,5 \%)$, et à aller sur Internet (11\% en 2008$)$, et relèvent donc également de cette famille des «nouveaux écrans ». Corrélativement, la convergence tendancielle des usages - les nouveaux écrans concentrent désormais l'essentiel des usages autrefois liés aux médias traditionnels, radio, télévision, écoute de musique enregistrée - favorise une polyvalence accrue des temps culturels et un accroissement des consommations simultanées : les plus jeunes sont souvent multitâches sur leurs ordinateur; et cette polyactivité "positive", qui n'est en rien du zapping, met à mal les catégories d'enquête (distinction entre activité principale et activité secondaire) et incite à repenser le modèle linéaire et unidimensionnel de la consommation culturelle.

Ce nouvel âge médiatique est également caractérisé par une hybridation des usages qui traversent les frontières et les interdictions habituelles: l'écriture de soi entre dans l'espace public des blogs, une émission de télévision interdite par les parents est regardée un peu plus tard sur l'ordinateur ou le smartphone... La désinstitutionalisation et l'individualisation des modes de consommation émancipation des grilles de programmes grâce au téléchargement, au podcast et au streaming, démultiplication des modalités de consommation grâces aux nouveaux médias - modifient en outre la place relative des offreurs et des consommateurs culturels ainsi que le rapport au temps et aux objets culturels des jeunes générations (temps linéaire des médias programmés versus temps cursif des hypermédias). Ces mutations incitent à repenser les catégories, souvent opposées, du producteur/créateur et du consommateur. En ce sens, l'ordinateur, pas plus qu'Internet, ne sont des médias, 
mais plutôt des hypermédias, réunissant les capacités des médias qui les ont précédés (son, image, texte), en y ajoutant une capacité d'action sur les modalités de réception comme sur les contenus que ne permettaient pas les précédents, si ce n'est à la marge : tout comme l'ordinateur, le portable devient ainsi un espace à soi, personnel et personnalisé (Allard, 2010), où la capacité médiatique révèle les compétences individuelles de ceux qui les utilisent, mais devient également caractéristique d'un groupe d'âge. C'est de ce sentiment de dépassement, à la fois technique et " éducatif ", sentiment accru par la rapidité des évolutions et l'extension des possibilités offertes par cet outil, que peuvent alors naître sinon de nouvelles "paniques morales ${ }^{5}$, du moins un préjugé de plus en plus répandu selon lequel la génération numérique se serait totalement affranchie, pour le meilleur ou pour le pire, de l'emprise culturelle parentale.

\section{La dynamique sociale et culturelle des loisirs numériques, entre transmission et innovation}

15 En matière de pratiques numériques peut-être encore plus que dans les autres domaines de la vie culturelle et des loisirs, il devient de plus en plus difficile de penser les processus de transmission d'une génération à la suivante comme des processus d'inculcation: les usages numériques des générations les plus récentes, s'ils sont plus importants et plus diversifiés chez les adolescents dont les parents sont eux-mêmes plus habitués à l'ordinateur, ne s'y conforment pas mécaniquement. Assiste-t-on en la matière à l'apparition de nouvelles formes de transmission culturelle, profondément marquées par un travail de transmutation des pratiques et des objets qui leur servent de supports?

\section{Un investissement générationnel inédit}

Que l'on compare leurs niveaux d'usages de l'ordinateur avec ceux de leurs parents, ou plus largement avec ceux des générations plus âgées au sein de la population française, on observe que les adolescents sont les plus impliqués dans ces nouvelles pratiques. Ainsi, dans l'enquête longitudinale, les parents déclarent en 2002 en moyenne moins de deux usages différents de l'ordinateur, alors que leur enfant en déclare 2,6 la même année, à la fois parce que les parents comptent dans leurs rangs une proportion plus grande de non-utilisateurs ( $8,5 \%$ des parents n'en ont aucun usage contre seulement $3 \%$ des enfants) et parce qu' ils ont des usages plus restreints que leurs enfants (18,5\% des parents déclarent plus de trois usages différents contre $48 \%$ des enfants la même année). Cette tendance est corroborée par l'enquête Pratiques culturelles des Français: $86 \%$ des 15-24 ans et encore $81 \%$ des $25-34$ ans utilisent un ordinateur à des fins de loisirs chaque jour, contre seulement $69 \%$ des $35-44$ ans et $56 \%$ des $45-54$ ans. De même, $91 \%$ des 15-19 ans ont utilisé Internet au cours du dernier mois, contre 78 \% des 25-34 ans et 52\% des 45-54 ans (Donnat, 2009 : 46-47). Les adolescents d'aujourd'hui sont, plus que les générations précédentes, porteurs de ce nouveau régime de valeur hypermédiatique, et le premier facteur de stratification des pratiques numériques semble donc bien être l'appartenance générationnelle. 


\section{Influences et transmissions}

Comment comprendre cet investissement générationnel ? Il doit évidemment aux effets de période : comment les générations antérieures auraient-elles pu être aussi investies que les plus jeunes, alors qu'elles sont issues d'un monde où le numérique était inexistant ou balbutiant? Mais l'analyse de cet investissement nouveau permet également d'interroger les modes d'influence et de transmission de cette appétence et de ces compétences numériques.

Les influences s'entremêlent et la dynamique des transmissions est subtile. Une modélisation ${ }^{6}$ de la fréquence d'utilisation de l'ordinateur lors de la première vague de l'enquête, à 11 ans, fait apparaître que dès cet âge l'influence majeure provient, toutes choses égales par ailleurs, des frères et sœurs, devant l'origine sociale et le sexe. Et l'observation des modes d'utilisation de l'ordinateur, entre accompagnement parental, fraternel, juvénile ou usages solitaires, indique combien l'avancée en âge au fil de l'adolescence accroît tendanciellement la part des influences juvéniles (Tableau 2): l'autonomie des usages, déjà acquise à la fin de l'école primaire, prend appui précocement sur les pairs : dès 15 ans, les copains sont les principaux pourvoyeurs d'influence. Dans ce contexte, le rôle de l'école est très secondaire, les apprentissages scolaires (type B2i) apparaissant en décalage avec les compétences et usages des préadolescents et adolescents.

Tableau : Accompagnement de l'usage de l'ordinateur et avancée en âge (\%)

\begin{tabular}{|l|l|l|l|l|}
\hline Utilise souvent un ordinateur... & $\mathbf{1 1}$ ans & $\mathbf{1 3}$ ans & $\mathbf{1 5}$ ans & $\mathbf{1 7}$ ans \\
\hline Avec le père ou la mère & 19 & 16 & 8 & 5 \\
\hline Avec un frère ou une sœur & 26 & 23 & 15 & 11 \\
\hline Avec un ou des copains, copines & 12 & 18 & 21 & 20 \\
\hline Seul(e) & 81 & 92 & 94 & 95 \\
\hline
\end{tabular}

Base : enfants qui utilisent un ordinateur au moins une fois par mois.

Note de lecture : Parmi les enfants de 11 ans qui utilisent l'ordinateur au moins une fois par mois, 19 \% déclarent le faire souvent avec leur père ou leur mère. Au même âge, $81 \%$ déclarent l'utiliser souvent seul.

On observe donc un basculement de l'univers familial vers l'univers juvénile qui correspond à l'autonomisation progressive de l'adolescent. Ce basculement n'est pas nouveau et apparaît consubstantiel à l'adolescence depuis qu'elle intéresse les sociologues (Galland, 2008, 2010, 2011). C'est son périmètre et sa forme qui sont plus originaux. Là où les générations précédentes entraient en opposition (les cultures jeunes comme contre-cultures), les jeunes générations entrent en cohabitation: les parents, anciens tenants des cultures jeunes des années 1970, ont fait leur l'idée selon laquelle chaque âge et chaque génération était porteuse d'une culture. Des expressions comme «c'est de son âge » ou encore "c'est leur culture » disent cette acceptation entre les générations, qui n'interdit pas les formes de transmission ascendante et descendante (Martin, 2007). 


\section{Transmission structurelle et mutations conjoncturelles}

20 L'exemple du jeu des influences concernant les pratiques numériques incite à réévaluer
ici la notion de transmission, à l'aune des mutations conjoncturelles qui en permanence
font évoluer le champ culturel (Octobre, Détrez, Mercklé \& Berthomier, 2011). Le passage d'une génération à l'autre porte les traces des mutations économiques, sociologiques, technologiques, culturelles, pédagogiques, etc. Ces mutations de la société sont des filtres entre ce qui est transmis et ce qui est hérité, transformant les objets au fil des générations (Lahaye, Pourtois \& Desmet, 2007). La transmission n'est donc pas la reproduction à l'identique de comportements d'une génération à une autre, mais de dispositions; il s'agit autrement dit d'un processus de conversion, qui mêle déconstruction, reconstruction, mutation et appropriation (Lahire, 1995). Il se comprend au travers des identités générationnelles, qui se donnent à lire à travers les comportements conjugaux (Kaufmann, 2008), procréatifs (Goss et Mehl, 2011), éducatifs ${ }^{7}$ et... culturels.

Ces identités constituent des rapports plus globaux au champ culturel : ainsi à chaque âge, les adolescents les plus investis globalement dans les loisirs culturels sont ceux dont les parents sont eux-mêmes les plus investis, quand bien même les objets ou pratiques culturelles que les uns et les autres choisissent ne sont pas identiques ${ }^{8}$. Plus qu'en termes de pratiques, les transmissions des dispositions culturelles se jouent en termes de représentations: à chaque âge, les adolescents les plus investis dans les loisirs culturels sont ceux dont les parents affectent le plus aux loisirs des objectifs d'épanouissement personnels. C'est particulièrement vrai dans le cas de la lecture, pratique par ailleurs rentable scolairement : les plus lecteurs sont les enfants de cadres. Les parents cadres déclarent en 2002 que la lecture sert l'épanouissement personnel (82\%) avant la réussite scolaire $(73,5 \%)$. Pour les parents ouvriers, la lecture est utilitaire avant tout $(81,5 \%$ disent qu'elle sert la réussite scolaire et $54,5 \%$ l'épanouissement personnel). S'agissant des pratiques numériques, dans un contexte marqué par des mutations technologiques très rapides, les parents sont moins à même d'enseigner des usages contextualisés (comment utiliser Facebook) que de transmettre des ressources dispositionnelles génériques (par exemple, des rapports à l'intimité et à l'exposition de soi) qui restent fortement différenciées suivant le milieu social ou le genre. Ici, plus probablement encore que dans les autres registres de loisirs, la socialisation est de moins en moins affaire d'accompagnement, autrement dit de transmission explicite, et de plus en plus affaire de climat familial, d'imprégnation, de socialisation « à distance ».

$\mathrm{Au}$ total, il s'agit donc non seulement d'une transformation de la structure du capital culturel et des usages d'une génération à l'autre - à chaque génération de familles correspondant un contexte socioculturel propre - mais aussi, plus fondamentalement, d'une mutation des processus de transmission : la société contemporaine se caractérise par une individualisation et une désinstitutionalisation relatives et la socialisation n'y est plus considérée comme l'adoption des normes d'un groupe mais comme le déploiement des moyens disponibles à l'individu pour se réaliser lui-même. Ce modèle, qui n'est sans doute pas moins normatif en réalité que le précédent, initialement caractéristique des classes favorisées, tend à se répandre dans l'ensemble de la société, 
et à imposer ses exigences dans les représentations des classes moyennes et populaires (de Singly, 2009).

\section{La recomposition de la stratification sociale des pratiques culturelles autour des loisirs numériques}

Pour autant, les classements ne disparaissent pas dans un espace social qui serait devenu atomisé : ce qui se transmet, ce n'est pas tant le volume absolu de ce capital culturel, que la position qu'il occupe dans l'espace symbolique et fait occuper dans l'espace social, dans un contexte socio-historique qui évolue d'une génération à l'autre. Les logiques de classement et de différenciation ne sont donc pas absentes de l'univers numérique, et elles ne sont pas sans rappeler celles qui sont à l'œuvre dans le domaine scolaire (Duru-Bellat \& Van Zanten, 2006) : la massification n'y est pas mécaniquement synonyme de démocratisation, et malgré la généralisation de l'équipement des foyers en ordinateurs et en accès à Internet, les pratiques numériques des adolescents restent différenciées selon leurs origines sociales, ainsi que selon leur sexe. Les enfants de milieux favorisés et de milieux populaires n'accèdent pas au même âge à l'utilisation quotidienne de l'ordinateur, n'ont pas la même maîtrise de ses usages connectés, en particulier des plus innovants; quand bien même ils y passeraient autant de temps, garçons et filles ne font pas la même chose devant l'ordinateur.

Tableau : Utilisation quotidienne de l'ordinateur et avancée en âge (\%)

\begin{tabular}{|l|l|l|l|l|}
\hline & 11 ans & 13 ans & 15 ans & 17 ans \\
\hline Ensemble & 14 & 26 & 57 & 69 \\
\hline Garçons & 16 & 26 & 57 & 72 \\
\hline Filles & 13 & 25 & 59 & 66 \\
\hline OR garçons/filles & 1,31 & 1,06 & 0,94 & 1,27 \\
\hline Enfants de cadres & 19 & 36 & 68 & 75 \\
\hline Enfants de professions intermédiaires & 15 & 30 & 65 & 72 \\
\hline Enfants d'employés & 14 & 27 & 56 & 70 \\
\hline Enfants d'ouvriers & 12 & 20 & 51 & 68 \\
\hline OR chefs de famille cadres/ouvriers & 1,74 & 2,21 & 2,07 & 1,42 \\
\hline Enfants de pères bacheliers & 19 & 33 & 67 & 73 \\
\hline Enfants de pères non bacheliers & 12 & 22 & 54 & 68 \\
\hline OR pères bacheliers/non bacheliers & 1,65 & $\mathbf{1 , 7 0}$ & $\mathbf{1 , 7 7}$ & $\mathbf{1 , 3 0}$ \\
\hline Enfants de mères bachelières & 16 & 31 & 66 & 70 \\
\hline
\end{tabular}




\begin{tabular}{|l|l|l|l|l|}
\hline Enfants de mères non bachelières & 13 & 22 & 53 & 68 \\
\hline OR mères bachelières/non bachelières & $\mathbf{1 , 2 5}$ & $\mathbf{1 , 6 4}$ & $\mathbf{1 , 7 7}$ & $\mathbf{1 , 1 0}$ \\
\hline
\end{tabular}

Base : tous les enfants.

Note de lecture : à 11 ans, $19 \%$ des enfants de cadres utilisaient un ordinateur tous les jours ou presque (et $81 \%$ moins souvent), contre $12 \%$ des enfants d'ouvriers (88\% le faisaient moins souvent). L'odds ratio (OR) enfants de cadres / enfants d'ouvriers était de $(19 \div 81) /(12 \div 88)=1,7$, ce qui signifie que les enfants de cadres avaient 1,7 fois plus de chances que les enfants d'ouvriers d'utiliser quotidiennement l'ordinateur plutôt que de l'utiliser moins souvent.

\section{L'utilisation de l'ordinateur : «Digital Natives » et « Digital Immigrants »}

La massification de l'utilisation de l'ordinateur est bien visible à la fin de l'adolescence de la cohorte suivie par l'enquête. En 2008, alors que les adolescents de l'échantillon avaient 17 ans, les variations d'intensité de l'utilisation de l'ordinateur imputables à l'origine sociale, si elles subsistent, apparaissent fortement atténuées : $75 \%$ des enfants de cadres utilisent un ordinateur tous les jours, contre $68 \%$ des enfants d'ouvriers (Tableau 3), et il n'y a guère de différences entre garçons et filles en termes de fréquence d'utilisation de l'ordinateur. De même, l'attachement à l'ordinateur est massif à 17 ans, quels que soient l'origine sociale et le genre : $78 \%$ des enfants de cadres et $73 \%$ des enfants d'ouvriers déclarent à cet âge que cela leur manquerait beaucoup s'ils ne pouvaient plus utiliser l'ordinateur ; c'est le cas également de $77 \%$ des garçons et $76 \%$ des filles.

Mais en réalité, ce que montre l'enquête longitudinale, c'est que cette relative convergence des fréquences d'utilisation et des attachements à l'ordinateur n'intervient qu'à l'orée de l'âge adulte, après une traversée de l'adolescence marquée par des différences beaucoup plus importantes: pendant pratiquement toute leur adolescence, les enfants de cadres avaient eu plus de deux fois plus de chances que les enfants d'ouvriers d'utiliser quotidiennement l'ordinateur. Et la même observation vaut si l'on considère non plus l'intensité d'utilisation mais l'attachement.

Tableau : Ancienneté de l'utilisation de l'ordinateur (\%)

\begin{tabular}{|l|l|l|l|l|l|l|l|l|l|}
\hline Garçons & Filles & Agriculteurs & $\begin{array}{l}\text { Artisans, } \\
\text { commerçants, } \\
\text { chefs } \\
\text { d'entreprise }\end{array}$ & $\begin{array}{l}\text { Cadres, } \\
\text { professions } \\
\text { intellectuelles }\end{array}$ & $\begin{array}{l}\text { Professions } \\
\text { intermédiaires }\end{array}$ & Employés & Ouvriers & Ensemble \\
\hline $\begin{array}{l}\text { Avant } \\
\begin{array}{l}\text { ans } \\
\text { ans }\end{array}\end{array}$ & 50 & 49 & 52 & 61 & 60 & 48 & 42 & 51 \\
\hline $\begin{array}{l}\text { Entre } \\
11 \text { et } \\
\begin{array}{l}\text { ans } \\
\text { ans }\end{array}\end{array}$ & 25 & 21 & 29 & 23 & 24 & 30 & 28 & 26 \\
\hline
\end{tabular}




\begin{tabular}{|l|l|l|l|l|l|l|l|l|l|}
\hline $\begin{array}{l}\text { Après } \\
13 \\
\text { ans }\end{array}$ & 20 & 21 & 29 & 16 & 15 & 16 & 21 & 27 & $\mathbf{2 1}$ \\
\hline Jamais & 2 & 1 & 1 & 2 & 1 & 1 & 1 & 3 & 2 \\
\hline Total & 100 & 100 & 100 & 100 & 100 & 100 & 100 & 100 & $\mathbf{1 0 0}$ \\
\hline
\end{tabular}

Base : tous les enfants.

Note de lecture : 49 \% des enfants d'agriculteurs ont utilisé l'ordinateur plusieurs fois par semaine pour la première fois avant 11 ans, et $21 \%$ entre 11 et 13 ans. Ancienneté et sexe : khi-2=3,1 p=0,38 (ns) ; ancienneté et PCS du père : khi-2=177,2 p<0,0001 (***).

26 Ce que la convergence à la fin de l'adolescence est susceptible de masquer, mais ne suffit pas à annuler, c'est donc l'ancienneté de la familiarité avec l'ordinateur, qui reste dans cette génération extrêmement variable selon le milieu social, alors qu'elle ne l'est absolument pas selon le sexe. À partir des fréquences d'utilisation de l'ordinateur à chaque vague de l'enquête, il est possible de construire une mesure de cette ancienneté, ici comprise comme l'âge auquel on a relevé pour la première fois une utilisation de l'ordinateur plusieurs fois par semaine (Tableau 4):

Alors que $61 \%$ des enfants de cadres et $60 \%$ des enfants de professions intermédiaires utilisaient déjà un ordinateur plusieurs fois par semaine à 11 ans, ce n'était le cas que de $42 \%$ des enfants d'ouvriers. La majorité d'entre eux va en fait découvrir cette utilisation pluri-hebdomadaire après 11 ans : entre 11 et 13 ans pour $28 \%$ d'entre eux, et même après 13 ans pour $27 \%$ d'entre eux (alors que cette découverte tardive, à la fin du collège et au début du lycée, ne concerne que $15 \%$ des enfants de cadres et $16 \%$ des enfants de professions intermédiaires). Indéniablement donc, le passage à une utilisation régulière de l'ordinateur est plus précoce chez les enfants de cadres. Et même si l'enquête ne débute qu'à 11 ans, une question posée aux parents permet tout de même de dire qu'elle est pour certains beaucoup plus précoce : à la question « Avant que votre enfant entre à l'école primaire, quelles activités faisiez-vous souvent avec lui (elle) ? », $39 \%$ des parents cadres mentionnent l'ordinateur, contre seulement $16 \%$ des ouvriers et $21 \%$ des employés...

Pour comprendre l'origine de ce décalage de calendrier, il faut revenir en réalité presque dix ans en arrière, au début de l'enquête : en 2002, au moment de la première vague de l'enquête, et alors que les enfants ont 11 ans et sont pour la plupart d'entre eux en $\mathrm{CM} 2$, les inégalités d'équipement en informatique étaient en effet encore très fortes: déjà $90 \%$ des enfants de cadres avaient un ordinateur à domicile, contre seulement $56 \%$ des enfants d'ouvriers; plus de $80 \%$ des parents bacheliers sont équipés, contre à peine $40 \%$ des parents non-diplômés. Et à cette fracture encore très importante en termes d'équipement, s'en ajoute une seconde, de moindre importance, en termes d'usages : même parmi les parents équipés, la familiarité avec l'ordinateur reste inégale. En réalité, la moitié des parents ouvriers ou employés équipés utilisent leur ordinateur moins d'une fois par semaine, contre seulement un tiers des parents cadres; inversement, $27 \%$ des cadres équipés l'utilisent tous les jours ou presque, contre seulement $20 \%$ des parents ouvriers et $18 \%$ des parents employés (ce sont plus souvent des femmes, et celles-ci ont une utilisation moins fréquente de l'ordinateur que leur conjoint). 
29 L'enfance et le début de l'adolescence de la cohorte suivie par l'enquête s'enracinent donc dans un contexte socio-technique marqué par de fortes disparités des taux de possession d'ordinateurs domestiques, qui font qu'en réalité une majorité des enfants de milieux favorisés pourraient être considérés comme des "digital natives», ou au moins des "early adopters", ayant accédé à une utilisation régulière de l'ordinateur avant l'entrée au collège, et parfois même avant l'entrée à l'école primaire, tandis qu'au contraire une majorité des enfants de milieux populaires seraient des "digital immigrants », qui n'y auraient accédé que plus tard, au collège ou au début du lycée.

\section{Le déplacement de la fracture numérique : différenciation et diversification des usages de l'ordinateur}

30 À l'entrée des enfants du panel dans l'adolescence, en 2002, leurs parents ne se différenciaient pas seulement par leur équipement, mais également par leurs usages de cet équipement. Dans l'ensemble, les enfants d'origine favorisée avaient des parents multi-utilisateurs, dont les usages nombreux combinaient fonctions professionnelles, utilitaires (comptes, recherches d'informations) et communicationnelles, tandis que les enfants d'origine populaire avaient des parents qui avaient un usage principalement récréatif de l'ordinateur domestique : $38 \%$ des parents ouvriers utilisent l'ordinateur pour jouer, contre seulement $25 \%$ des parents cadres, et c'est le seul type d'usage de l'ordinateur plus fréquemment observé chez les premiers que chez les seconds.

31 Ces différences vont se retrouver dans les types d'usages des adolescents eux-mêmes, mais d'une façon qui peut sembler de prime abord paradoxale : les enfants d'ouvriers restent longtemps (jusqu'à 15 ans) beaucoup plus nombreux que les enfants de cadres à déclarer à un usage exclusivement scolaire de l'ordinateur ( $21 \%$ contre $13 \%$ à 13 ans, encore $14 \%$ contre $7 \%$ à 15 ans), tandis que les seconds sont plus nombreux que les premiers à en déclarer un usage exclusivement ludique ( $36 \%$ contre $31 \%$ à 13 ans); les enfants de cadres sont du reste plus nombreux que tous les autres à se servir de l'ordinateur pour jouer à des jeux vidéo. Ce n'est pas tant que les enfants des classes populaires utilisent plus que les autres leur ordinateur pour l'école : c'est surtout qu'ils l'utilisent moins pour jouer, ce qui s'explique en bonne partie par le fait qu'en revanche ils utilisent nettement plus souvent une console de jeu connectée à la télévision, cette coupure entre jeu sur ordinateur et jeux sur console selon l'origine sociale étant du reste attestée depuis longtemps (Bruno, 1993; Trémel, 1999). Dans les familles populaires, les parents utilisent l'ordinateur principalement pour jouer, et leurs enfants l'utilisent pour faire leurs devoirs, la console de jeux étant dévolue à la fonction ludique. Dans les familles dont les parents sont cadres ou professions intermédiaires, moins équipées en consoles de jeu mais étant plus fréquemment confrontées à l'apprentissage de l'informatique au travail (Gollac \& Kramarz, 2000), l'ordinateur sert beaucoup plus fréquemment à des usages mixtes, et donc plus diversifiés. 
Figure : Les usages connectés et l'avancée en âge (\%)

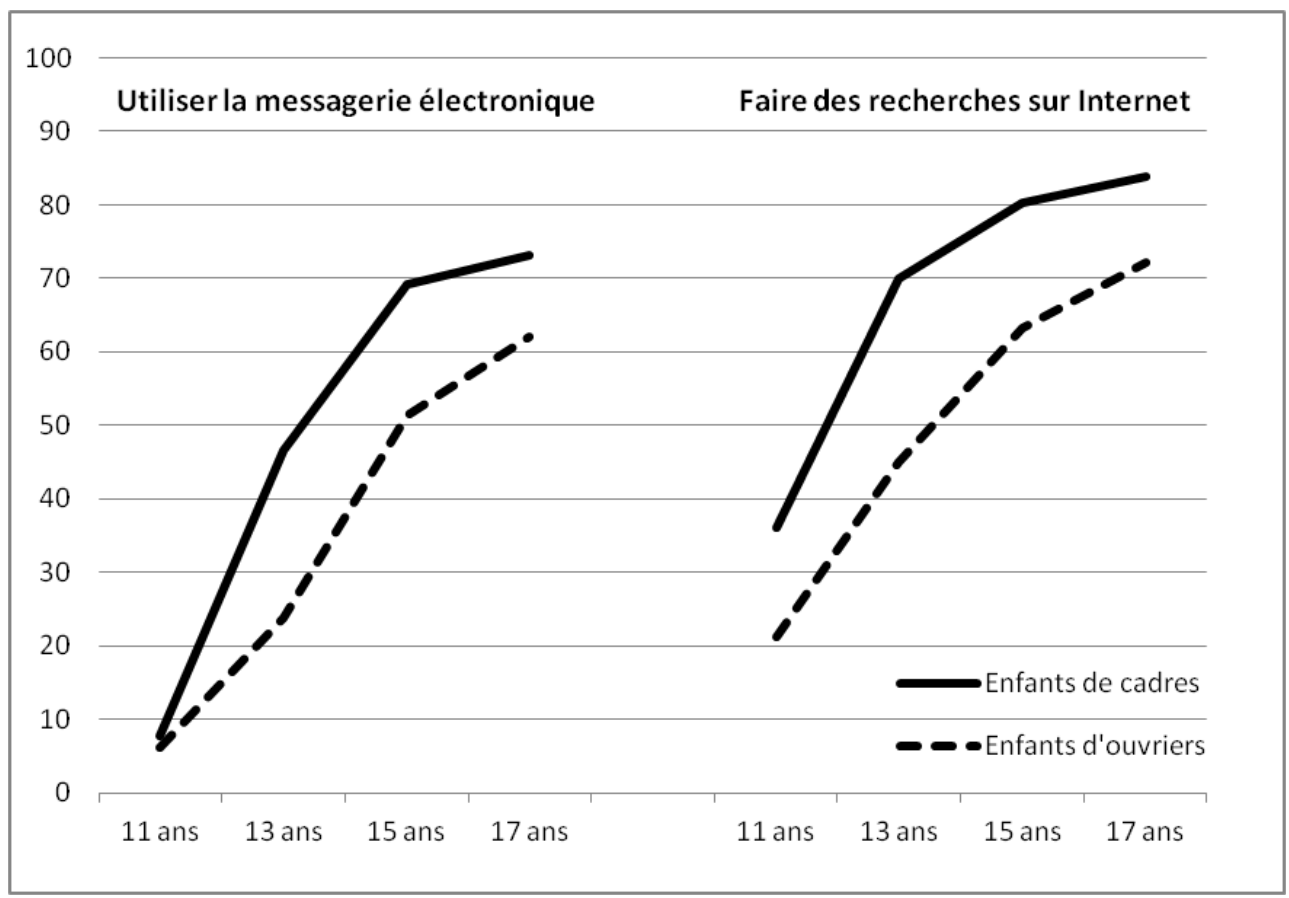

Base : Enfants qui utilisent l'ordinateur au moins une fois par mois

Note de lecture : A 11 ans, 8 \% des enfants de cadres utilisaient l'ordinateur pour envoyer des messages électroniques.

Cet « éclectisme» (Peterson, 1992 ; Peterson \& Kern, 1996) numérique des adolescents des milieux favorisés est encore renforcé par leur plus grande familiarité avec l'Internet. Là encore, la comparaison avec la dynamique de la massification scolaire est éclairante : avec la généralisation de l'ordinateur domestique, le terrain de jeu de la distinction numérique s'est déplacé vers la maîtrise des NTIC, des usages connectés de l'ordinateur. Et à 17 ans, alors que les équipements et les fréquences d'utilisation ont largement convergé, les usages connectés restent encore significativement plus fréquents chez les adolescents de milieux favorisés : ils sont toujours plus nombreux que les enfants d'ouvriers à utiliser la messagerie électronique et faire des recherches sur Internet (Figure 2), mais aussi à écouter de la musique, regarder des vidéos et pratiquer le téléchargement. Tout se passe comme si les adolescents de milieux favorisés faisaient ainsi preuve, dans ces différents domaines, d'une familiarité avec les nouveaux usages numériques qui a sûrement à voir avec l'ancienneté de l'utilisation de l'ordinateur, et qui est probablement aussi l'expression d'une compétence transversale assimilable à ce qu'en anglais on appelle la « digital literacy ( (Gilster, 1997) et que les Québécois traduisent par "littératie numérique ». Au-delà de ces mesures des différences d'usages qui restent très générales, d'autres travaux récents ont d'ailleurs souligné que malgré un accès au réseau là encore de plus en plus massif, l'illégitimité persistante de certaines formes d'expression sur Internet (qui a du reste contribué à un retour inattendu de l'écrit comme forme dominante d'expression) continue de tenir les classes populaires à l'écart des normes d'autonomie, d'accomplissement de soi et de reconnaissance imposées dans ces nouveaux espaces publics par les classes dominantes (Granjon, 2009). 

l'Internet plus répandus, une approche moins strictement scolaire de l'ordinateur, une familiarité plus précoce avec les différents usages connectés - pour marquer au sceau de la diversification des usages (informationnels, ludiques, communicationnels) beaucoup plus nettement l'expérience numérique des adolescents des classes favorisées que celles des adolescents des milieux populaires - et cela même jusqu'à l'entrée de cette cohorte dans l'âge adulte.

Cette diversification des usages avec l'avancée en âge, qui caractérise plus fréquemment les enfants de cadres que les enfants d'ouvriers, a clairement pour moteur une adoption plus précoce des nouveaux usages numériques par les premiers que par les seconds. Bien sûr, il est possible de faire l'hypothèse qu'avec la généralisation de l'équipement des foyers en ordinateurs et en accès à Internet, le « retard » invoqué jusque-là pour expliquer ces inégalités devrait cesser de produire ses effets, et donc de faire l'hypothèse qu'une progressive égalisation des compétences et des usages devrait suivre l'égalisation des équipements. Mais ce serait oublier le mécanisme classique (Goblot, 1967) selon lequel les attributs de la compétence, et donc de la distinction numériques sont en perpétuel déplacement : même si la méthodologie de l'enquête longitudinale dans un contexte d'innovation rapide se prête parfois mal à la mesure de cette dynamique différentielle d'adoption des innovations, il est évident que dans le domaine des nouveaux usages numériques, les enfants de milieux favorisés devancent toujours les enfants de milieux populaires. C'est le cas au moment de la transformation de l'ordinateur connecté en appareil multimédia ; et c'est encore le cas à la toute fin de l'enquête, lors de la dernière vague, avec l'apparition de Facebook: en 2008, alors que le réseau social américain commence à peine à percer en France, déjà $4 \%$ des enfants de cadres déclarent en être des utilisateurs réguliers, alors que ce n'est encore le cas d'aucun des enfants d'ouvriers du panel... Il faudrait de nouvelles enquêtes pour le mesurer précisément, mais il ne fait guère de doute que ce schéma s'est depuis reproduit autour de chacun des équipements et des usages émergents, et qu'il y a beaucoup plus d'adolescents de classes favorisées que de classes populaires parmi ceux qui ont remplacé ensuite Facebook par Google+, ou parmi les premiers utilisateurs des smartphones, et désormais des tablettes tactiles.

\section{Garçons et filles devant l'ordinateur}

Les résultats les plus généraux de l'enquête longitudinale sur les pratiques culturelles des adolescents (Octobre, Détrez, Mercklé \& Berthomier, 2010) montrent que les intensités de leurs consommations culturelles, en tout cas quand ils ne sont pas devant l'ordinateur, varient beaucoup plus en fonction du sexe que de l'origine sociale. Certains clichés voudraient qu'il en soit de même en matière de pratiques numériques, et que la stratification sociale du temps passé devant l'ordinateur soit aussi une affaire de genre : la représentation commune de la figure du geek, de l'adolescent passant ses journées et ses nuits rivé à sa machine, est généralement masculine. C'était peut-être vrai pour la génération précédente, socialisée à une informatique valorisant plus la virtuosité technique que la convivialité des usages : en 2002, au début de l'enquête, $28 \%$ des pères utilisaient leur ordinateur tous les jours ou presque, contre seulement $19 \%$ des mères, et à l'inverse, seulement un tiers des pères l'utilisaient moins d'une fois par semaine, contre près de la moitié des mères ; c'est encore le cas en partie pour les 30-45 
ans interrogés lors de la dernière enquête sur les pratiques culturelles des Français (Donnat, 2009)...

Mais ce n'est pas vrai, ou plus vrai, pour les enfants de notre cohorte : à 11 ans, $16 \%$ des garçons et $13 \%$ des filles utilisaient un ordinateur tous les jours, à 13 ans, il n'y avait aucune différence observable, et à 15 ans les utilisatrices quotidiennes de l'ordinateur étaient même un peu plus nombreuses (59 \%) que les garçons (57 \%) (Tableau 3). De ce fait, il n'y a pas d'adoption de l'utilisation quotidienne de l'ordinateur plus précoce chez les garçons que les filles: une petite moitié des filles comme des garçons ont commencé à utiliser l'ordinateur quotidiennement avant 11 ans, et l'autre moitié plus tardivement (Tableau 4). La " fracture numérique », au moins de ce point de vue, n'est donc pas d'abord une fracture genrée, mais bien une " fracture de classe ». Plus encore : si les enfants de cadres devancent les enfants d'ouvriers sur la voie de la diversification des usages, en revanche le fait d'être un garçon ou une fille ne fait en matière d'éclectisme numérique que très peu de différences.

Formellement, garçons et filles semblent donc égaux devant l'ordinateur, qui vient même parfois remettre en cause certains clivages habituels, car il est également susceptible de les rassembler autour d'usages communs : pour les unes comme pour les autres, la messagerie instantanée arrive ainsi en tête des usages de l'ordinateur à 17 ans. Souvent vu comme l'accès des filles à un outil technique, l'ordinateur propose aussi aux garçons une entrée dans la culture de la chambre (Glévarec, 2010), jusque-là fortement connotée par le féminin (Sefton-Green, 1994 ; Mcrobbie, 1991), tandis qu'eux étaient en quelque sorte assignés à l'extérieur du domicile: la "chambre digitale " ("digital bedroom», Sefton-Green, 1998) dote les garçons de compétences et appétences à l'égard de cette culture de chambre féminine, qu'objective la pratique du téléphone: les nouvelles technologies permettent aux garçons des usages conversationnels parfois inexistants sur leurs autres scènes sociales.

Mais la remise en cause de ces clivages traditionnels est loin d'être complète: en matière d'usages numériques, les distinctions de genre restent encore plus fortes que celles liées à l'origine sociale. Si à 17 ans, comme on l'a vu plus haut, les usages numériques principaux des enfants de milieux favorisés et de milieux populaires se sont rapprochés, en revanche les différences selon le sexe restent beaucoup plus marquées. Tout d'abord, si $42 \%$ des garçons utilisant un ordinateur le font encore exclusivement pour leurs loisirs, ce n'est plus le cas que de $24 \%$ des filles. La tendance s'inverse quand il s'agit de déclarer des usages à la fois scolaires et divertissants, les filles se montrant nettement plus polyvalentes que les garçons (55\% des garçons, $73 \%$ des filles). Si les garçons, dès l'enfance, jouent à des jeux vidéo sur ordinateur bien plus souvent que les filles, ils continuent à le faire à 17 ans (61\% des garçons contre $23 \%$ des filles), alors qu'elles s'en sont majoritairement détournées. Les garçons sont plus présents sur les forums, et téléchargent davantage de films; quant aux filles, elles privilégient le dessin ou les photos (seulement $18 \%$ des garçons, contre $36 \%$ des filles), la messagerie instantanée et la messagerie électronique, les recherches sur Internet, l'écoute et le téléchargement de musique. Au total donc, les filles et les garçons ne font pas la même chose devant l'ordinateur. Les unes et les autres, au moins jusqu'à 15 ans, en ont des usages aussi fréquents et diversifiés, mais ils se distinguent par des usages différents, qui renvoient à des principes de différenciation à l'œuvre aussi quand l'ordinateur est éteint: aux filles les usages plutôt scolaires, communicationnels et créatifs, aux garçons les usages plutôt récréatifs et techniques. 
39 Cela dit, il se pourrait bien aussi qu'à ces différences durables d'usages s'ajoute la réapparition de différences de niveaux de pratiques à la grande adolescence : à 17 ans, lors de la dernière vague de l'enquête, les utilisateurs quotidiens étaient redevenus significativement plus nombreux parmi les garçons (77\%) que parmi les filles (66 \%). Des différences d'équipement, qui n'existaient pas auparavant, surgissent parallèlement : à la fin de l'adolescence, les garçons se mettent en effet à avoir plus souvent que les filles un ordinateur qui leur est réservé ( $40 \%$ des garçons contre $30 \%$ des filles), tandis que les filles doivent plus fréquemment que les garçons le partager avec leurs frères et sœurs. Cette dernière différence rappelle celle observée au même moment chez les 20-45 ans en couple par l'enquête Pratiques culturelles des Français : les hommes y apparaissent plus souvent comme "utilisateurs principaux ou exclusifs ", et les femmes comme "utilisatrices secondaires» de l'ordinateur domestique (Donnat, 2009 : 54-55), ce qui laisse penser que cette asymétrie est donc transgénérationnelle.

\section{Conclusion}

L'utilisation de l'ordinateur, désormais massive, s'est clairement installée au centre d'un espace des styles de vie des adolescents qui reste dans son ensemble marqué par de profondes logiques de différenciation, voire d'opposition, en particulier entre pratiques « culturelles » et pratiques de « loisirs » ou de « divertissement », mais aussi entre activités domestiques et activités à l'extérieur. Cette "massification » de l'utilisation de l'ordinateur n'est pas pour autant synonyme de "démocratisation ", mais s'accompagne en réalité d'un déplacement de la fracture numérique : alors que les premières mesures communément admises de celle-ci, il y a une dizaine d'années (c'est-à-dire au début de notre enquête) se basaient effectivement sur les taux de possession d'un ordinateur, la définition désormais adoptée par la plupart des institutions internationales porte sur les inégalités d'accès aux TIC et plus précisément à Internet.

41 Or, la cohorte d'adolescents que nous avons observée apparaît clairement au cœur de cette transformation de la fracture numérique. Avec l'avancée en âge, les adolescents des années 2000 se différencient de moins en moins par la possession et la fréquence d'utilisation d'un ordinateur; en revanche, ils continuent de se différencier de façon significative par leurs usages de celui-ci, et en particulier par une plus grande familiarité des adolescents des milieux favorisés avec les usages connectés. De façon générale, puisqu'ils cumulent désormais les usages connectés et les usages non connectés de l'ordinateur, les adolescents des milieux favorisés apparaissent du coup comme faisant preuve, en matière de pratiques numériques aussi, d'une propension plus forte que les adolescents des milieux populaires à cet «éclectisme » que la sociologie de la culture regarde depuis une vingtaine d'années comme la nouvelle marque de fabrique de la « distinction ».

42 Au total, il n'est donc pas possible de désigner la cohorte des adolescents des années 2000 comme la première génération de "digital natives " sans commettre un abus de langage. Elle reste en réalité profondément partagée entre: d'une part une forte minorité de garçons et de filles effectivement familiarisés très tôt à l'utilisation de l'ordinateur (avant le primaire pour un nombre relativement important d'entre eux) et habitués à la fin de l'adolescence à des usages extrêmement diversifiés de l'outil informatique, et en particulier de l'Internet, les filles en ayant cependant des usages 
plus scolaires, communicationnels et créatifs, et les garçons des usages plus récréatifs et techniques; et d'autre part une majorité d'adolescents dont la familiarisation avec l'ordinateur est plus tardive, et date plutôt du collège, voire du début du lycée, et dont l'utilisation des nouveaux outils de communication reste encore, à l'entrée dans l'âge adulte, moins régulière et moins diversifiée.

Si enfin on ajoute que les adolescents de milieux favorisés sont enclins à adopter plus rapidement que les autres les usages numériques émergents, il faut probablement admettre que le déplacement continu des frontières du territoire de la "littératie numérique " va condamner une partie importante des prochaines cohortes d'adolescents de milieux populaires à rester des «digital immigrants ».

\section{BIBLIOGRAPHIE}

ALLARD Laurence (2010). Mythologie du portable, Paris, Cavalier bleu

BOURDIEU Pierre (1979). La distinction. Critique sociale du jugement, Paris, Ed. de Minuit.

BRUNO Pierre (1993). Les jeux vidéo, Paris, Syros.

BUCKINGHAM David (2000). After the Death of Childhood: Growing Up in the Age of Electronic Media, Cambridge, Polity Press.

DAGNAUD Monique (2011). Génération Y. Les jeunes et les réseaux sociaux, de la dérision à la subversion, Paris, SciencesPo.

DE SINGLY François (2002). « À quoi sert la famille? », in Dortier Jean-François (dir.), Familles, permanence et métamorphoses, Auxerre, Sciences Humaines.

DE SINGLY François (2009). Comment aider l'enfant à devenir lui-même ?, Paris, Armand Colin. DONNAT Olivier (1998). Les pratiques culturelles des Français. Enquête 1997, Paris, Ministère de la Culture, Documentation française.

DONNAT Olivier (1999). « La stratification sociale des pratiques culturelles et son évolution 1973-1997 », Revue Française de Sociologie, 40 (1), pp. 111-119.

DONNAT Olivier (2009). Les pratiques culturelles des Français à l'ère numérique, Paris, La Découverte / Ministère de la Culture et de la Communication.

DONNAT Olivier \& Daniel COGNEAU (1990). Les pratiques culturelles des Français. 1973-1989, Paris, Ministère de la Culture et de la Communication, La Découverte, Documentation française. DURU-BELlat Marie \& VAn ZANTEn Agnès (2006 [1992]). Sociologie de l'école, Paris, Armand Colin, 3ème édition.

FROW John (1995). Cultural studies and cultural value, Oxford, Oxford University Press.

GALLAND Olivier (2008). « Une nouvelle adolescence », Revue française de sociologie, 49 (4), pp. 819-826.

GALLAND Olivier (2010). « Une nouvelle classe d'âge ? ", Ethnologie française, 40 (1), pp. 5-10. 
GALLAND Olivier (2011 [1997]). Sociologie de la jeunesse, Paris, Armand Colin, 5ème édition.

GILSTER Paul (1997). Digital literacy, New York, Wiley.

GLÉVAREC Hervé (2010). La culture de la chambre. Préadolescence et culture contemporaine dans l'espace familial, Paris, La Documentation française.

GOBLOT Edmond (1967 [1925]). La barrière et le niveau. Etude sociologique sur la bourgeoisie française moderne, Paris, Presses Universitaires de France, Alcan.

GOLLAC Michel \& KRAMARZ Francis (2000). «L'informatique comme pratique et comme croyance », Actes de la recherche en sciences sociales, 134, pp. 4-21.

Goss Martine \& MEHL Dominique (2011). « Homoparentalités et gestation pour autrui », Enfance,

Familles, générations, 14, pp. 95-112.

GRANJON Fabien (2009). «Inégalités numériques et reconnaissance sociale. Des usages populaires de l'informatique connectée », Les Cahiers du numérique, 5 (1), pp. 19-45.

KAUFMANN Jean-Claude (2008). Quand Je est un autre, Paris, Armand Colin.

KREDENS Elodie \& FONTAR Barbara (2010). Comprendre le comportement des enfants sur Internet pour les prévenir des dangers, Fréquence Ecoles, Fondation pour l'Enfance.

LAHAYE Willy, POURTOIS Jean-Pierre \& DESMET Hughette (2007). Transmettre. D'une génération à l'autre, Paris, Presses universitaires de France.

LAHIRE Bernard (1995). Tableaux de familles. Heurs et malheurs scolaires en milieux populaires, Paris, Gallimard, Seuil.

LEHALLE Evelyne \& Lucien MIRONER (1993). Musées et visiteurs, un observatoire permanent des publics, Paris, Direction des musées de France.

MARESCA Bruno, PICARD Romain \& PILORIN Thomas (2011). « Dépenses culture-médias des ménages en France au milieu des années 2000 : une transformation structurelle », Culture Etudes, 2011-3, www2.culture.gouv.fr/culture/deps/2008/CE-2011-3-site.pdf

MARTIN Olivier (2007). « La conquête des outils électroniques de l'individualisation chez les 12-22 ans », Réseaux, 45, pp. 335-366.

MCROBBIE Angela (1991). Feminism and Youth Culture: From "Jackie" to "Just Seventeen", Cambridge, Unwin Hyman.

MERCKLÉ Pierre (2011 [2004]). Sociologie des réseaux sociaux, Paris, La Découverte, 3ème édition. METTON-GAYON Céline (2009). Les adolescents, leur téléphone et Internet. "Tu viens sur MSN ?", Paris, L'Harmattan.

OCTOBRE Sylvie (2004). Les loisirs culturels des 6-14 ans, Paris, La Documentation française. OCTOBRE Sylvie, DÉTREZ Christine, MERCKLÉ Pierre \& BERTHOMIER Nathalie (2010). L'enfance des loisirs. trajectoires communes et parcours individuels de la fin du primaire aux années lycée, Paris, La Documentation française.

OCTOBRE Sylvie, DÉTREZ Christine, MERCKLÉ Pierre \& BERTHOMIER Nathalie (2011). « La transmission culturelle : quelques éléments de réflexion à partir d'une enquête longitudinale sur les pratiques culturelles des adolescents ", Recherches familiales, 8, pp. 71-80.

PASQUIER Dominique (2005). Cultures lycéennes. La tyrannie de la majorité, Paris, Autrement. 
PETERSON Richard A. (1992). « Understanding Audience Segmentation : From Elite and Mass to Omnivore and Univore », Poetics, 21, pp. 243-258.

PETERSON Richard A. \& KERN R. M. (1996). « Changing highbrow taste : from snob to omnivore », American Sociological Review, 61 (5), pp. 900-907.

PRENSKY Mark (2001). « Digital Natives, Digital Immigrants », On the Horizon, 9 (5), pp. 1-6.

SEFTON-GREEN Julian (1994). Cultural Studies Goes to School, London, Taylor \& Francis.

SEFTON-GREEN Julian (1998). « Digital Visions: Children's Creative Uses of Digital Technologies », in Julian Sefton-Green \& David Buckingham (dir.), Digital Diversions: Youth Culture in the Age of Multimedia, London, Routledge, pp. 62-83.

SÉGALEN Martine, LAPIERRE Nicolas \& ATTIAS-DONFUT Claudine (2002). Le nouvel esprit de famille, Paris, Odile Jacob.

TRÉMEL Laurent (1999). « Les “jeux vidéo" : un ensemble à déconstruire, des pratiques à analyser », Revue Française de Pédagogie, 136, pp. 147-157.

WATKINS Craig (2009), The Young and the Digital, Boston, MA, Beacon.

\section{NOTES}

1. Les auteurs tiennent à remercier Nathalie Berthomier et Christine Détrez, qui ont réalisé cette enquête avec eux, et dont les analyses fournissent une part très importante de la matière sur laquelle s'appuie cet article. Ils remercient également Samuel Coavoux, Sébastien François et les relecteurs anonymes pour leurs nombreuses et très utiles remarques.

2. Les enquêtes sur les pratiques culturelles des Français portent sur des échantillons représentatifs de la population résidant en France et âgée de 15 ans et plus.

3. Dans le domaine des loisirs numériques, l'offre d'équipements et de services a évolué si vite qu'au cours de cette décennie 2000, on a effectivement pu parler de "révolution »: c'est le temps, vécu pratiquement « en direct » au fur et à mesure de l'avancée de l'enquête, de l'entrée dans "l'ère numérique » (Donnat, 2009), qui oblige l'enquête longitudinale à recomposer à chaque vague un équilibre, toujours provisoire, entre prise en compte des mutations et préservation de la comparabilité. Si certaines questions (par exemple sur les supports utilisés pour écouter de la musique) ont ainsi dû être reformulées, et si certains usages nouveaux ont pu être sous-estimés (par exemple l'explosion des réseaux sociaux en ligne, qui n'intervient que vers la fin de l'enquête), le caractère extensif du questionnaire a cependant permis de suivre de façon globalement cohérente les transformations des usages numériques des adolescents au cours de cette décennie.

4. 75\% des 13-17 ans possèdent un blog, 55\% des 11-13 ans ont une page Facebook (baromètre «Enfant et Internet ", Calysto, 2010) et 88\% des 15-17 ans ont des usages avancés (internet mobile, applications, appareil photo, radio, vidéo, mp3...) du téléphone portable (étude Médiamétrie-Nielsen, juillet-septembre 2009).

5. Ces paniques morales, comme le souligne Olivier Donnat (2009: 73), se sont déportées de la télévision aux nouveaux écrans : la télévision s'est banalisée, comme en témoigne la baisse dans la population française des réticents à son égard.

6. Un modèle de régression logistique a été construit avec les variables suivantes: PCS de la personne de référence du ménage, sexe de l'enfant, situation vis-à-vis de l'emploi (les deux parents travaillent, un seul travaille, aucune ne travaille), type de famille (classique, recomposée, monoparentale, autre), climat familial, taille de l'unité urbaine, classification de l'école en ZEP, 
diplômes du père et de la mère, type de socialisation culturelle de la fratrie, du groupe des pairs et de l'école. Pour plus de détails, voir Octobre, Détrez, Mercklé \& Berthomier, 2010 : 245.

7. Une comparaison des pratiques éducatives de trois générations montre ainsi une évolution des modèles éducatifs vers des formes plus égalitaires (Ségalen, Lapierre \& Attias-Donfut, 2002), ce qui redéfinit les postures parentales (De Singly, 2002).

8. On a construit un score global de participation culturelle pour les parents et pour leur enfant à chaque âge.

\section{RÉSUMÉS}

La révolution numérique ne semble pas avoir changé les liens étroits entre position sociale d'une part, et dotation en équipements, détentions de compétences, intensités d'investissement dans les loisirs culturels, types d'usages et de préférences d'autre part. Malgré l'apparente généralisation des technologies numériques, d'importantes inégalités subsistent en matière aussi bien d'équipement des foyers en ordinateurs, que d'accès et d'usages de l'Internet. Qu'en sera-t-il des générations nées avec le numérique, qui ont été enfants et adolescents dans les années 2000 ? Nous nous proposons d'apporter des éléments de réponses à partir des résultats de l'enquête longitudinale sur les pratiques culturelles et de loisirs des enfants et des adolescents réalisée à l'initiative du Ministère de la Culture : 4000 enfants entrés en CP en 1997 et ayant grandi dans la décennie 2000, entre la généralisation du téléphone portable et celle des réseaux sociaux en ligne, ont été interrogés tous les deux ans par questionnaire sur leurs loisirs, leurs pratiques culturelles et leurs goûts entre 2002 et 2008. En nous concentrant sur les relations entre les pratiques numériques (en termes de fréquences et types d'usages) de cette génération d'adolescents et leurs transformations d'une part, et les équipements et les pratiques des parents d'autre part, nous entendons discuter l'idée d'un rapport unifié des adolescents à la culture numérique, et en proposer un tableau qui tienne mieux compte à la fois de la complexité et de la diversité des usages, et des clivages sociaux qui continuent de les traverser, ainsi que de la recomposition de ces clivages avec l'avancée en âge chez les filles et chez les garçons.

The digital revolution may not have changed the tight link between social status, on the one hand, and equipment rates, skills, participation in cultural leisures, types of uses, and taste, on the other. Despite the seemingly large spread of digital technologies, substantial inequalities remain in terms of computer equipment as well as access and use of the Internet. What about the digital generation, i.e. the individuals who were children or teenagers during the 2000s? This article relies on an extensive longitudinal study of the cultural and leisure activities of children and teenagers, set up by the French Ministry of Culture. 4000 children who entered the Preparatory Course (first year of elementary education) in 1997, and have consequently witnessed the diffusion of mobile phones and online social networks, were surveyed every two years between 2002 and 2008. The questions dealt with their leisures, cultural activities, and tastes.

We focus on the relations between the digital activities of this generation, and their transformations on the one hand, and between the equipment and the activities of their parents on the other hand. We aim at qualifying the idea that teenagers of that particular generation have a unique relationship to digital culture. It is necessary to take into account both the 
complexity and the variety of uses, as well as the social inequalities that still prevail - although those inequalities tend to change while the teenagers, boys and girls, are aging.

INDEX

Mots-clés : culture, adolescence, pratiques numériques, Internet, stratification sociale

Keywords : culture, adolescence, digital uses, Internet, social stratification

\section{AUTEURS}

\section{PIERRE MERCKLÉ}

Ecole normale supérieure de Lyon, Centre Max Weber

\section{SYLVIE OCTOBRE}

Département des études, de la prospective et des statistiques du ministère de la culture et de la communication 